Provided for non-commercial research and education use. Not for reproduction, distribution or commercial use.

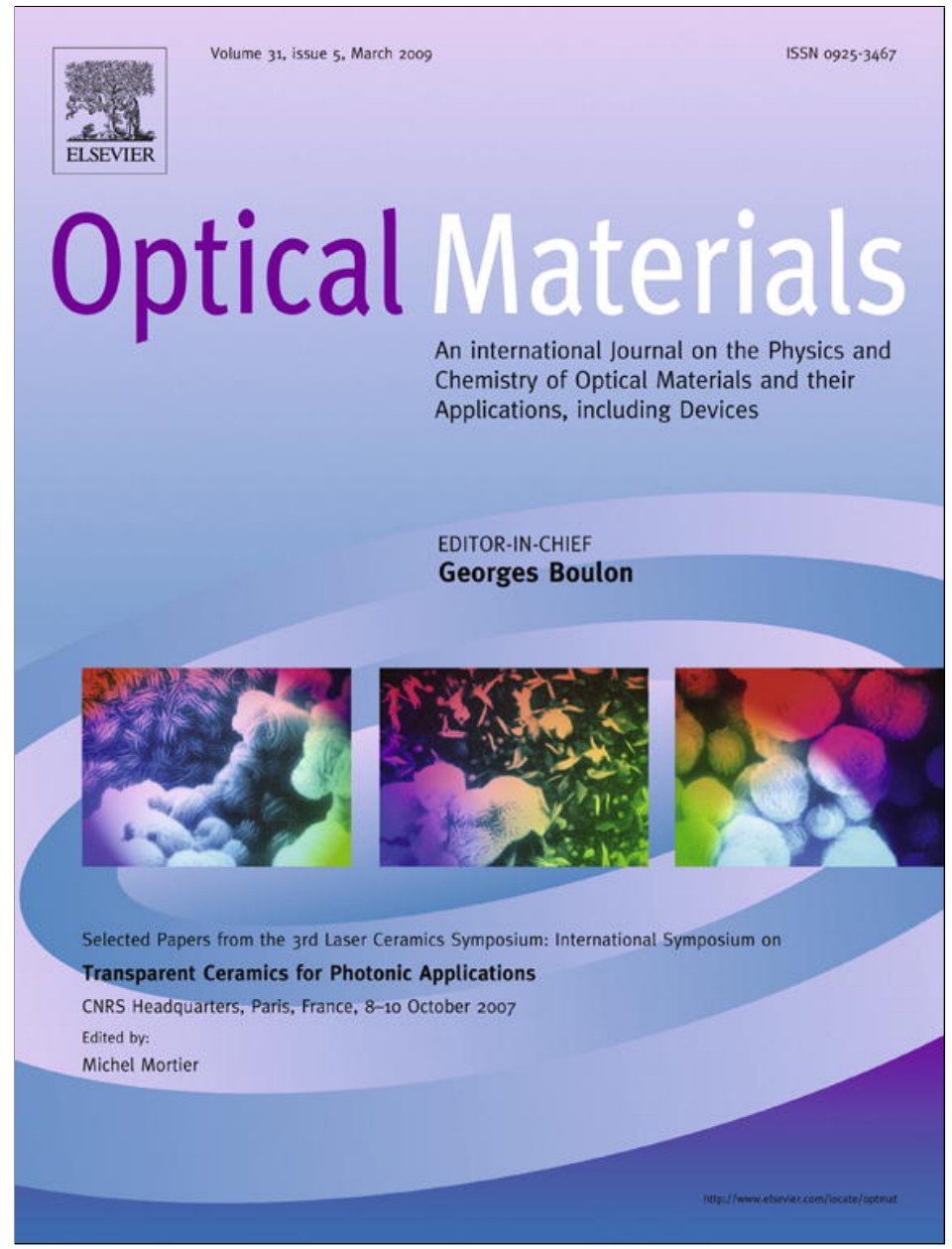

This article appeared in a journal published by Elsevier. The attached copy is furnished to the author for internal non-commercial research and education use, including for instruction at the authors institution and sharing with colleagues.

Other uses, including reproduction and distribution, or selling or licensing copies, or posting to personal, institutional or third party websites are prohibited.

In most cases authors are permitted to post their version of the article (e.g. in Word or Tex form) to their personal website or institutional repository. Authors requiring further information regarding Elsevier's archiving and manuscript policies are encouraged to visit:

http://www.elsevier.com/copyright 


\title{
Scaling laws of disk lasers
}

\author{
Dmitrii Kouznetsov*, Jean-François Bisson, Kenichi Ueda \\ Institute for Laser Science, University of Electro Communications, Chofu, Tokyo 182-8585, Japan
}

\section{A R T I C L E I N F O}

\section{Article history:}

Received 18 December 2007

Received in revised form 18 March 2008

Accepted 18 March 2008

Available online 21 May 2008

\begin{abstract}
A B S T R A C T
Limit of power from amplified spontaneous emission (ASE), round-trip loss, and overheating is considered within simple model. The optimization of the output power with respect to size of the pumped region, its thickness and round-trip gain leads to the scaling laws for these parameters. In vicinity of the limit of power scaling, the round-trip loss should scale inversely proportional to the cubic root of the desired output power. This prediction agrees with the experimental data. The use of the thick undoped layer (anti-ASE cap) allows to increase for an order of magnitude the maximal power achievable at the same round-trip loss.
\end{abstract}

(c) 2008 Elsevier B.V. All rights reserved.

\section{Introduction}

Disk lasers [1-11] are believed to allow power scaling. This scaling could be limited by various mechanisms. In order to understand, which mechanism is dominant, we need at least qualitative estimates for each of them. The dominant mechanism should determine the scaling laws for the most important parameters of a powerful laser. In this paper, we consider the only one of possible mechanisms which may affect the power scaling. We analyze the limit which comes from the amplified spontaneous emission (ASE), overheating and round-trip loss. We use the simplest model [12] which still takes into account these three effects and allows to estimate the maximal power achievable at given value of the round-trip loss. In paper [13], the formalism is adopted for the case with an undoped layer (Fig. 1), which acts as anti-ASE cap [6]. Such generalization, as well as the estimates of the effective path of ASE, caused discussions, even in the case without anti-ASE cap [14].

In this paper we simplify the deduction and combine results of papers [12] and [13], making the most of the formulas valid for both cases, with the anti-ASE cap and without (Fig. 1a and b). Also, we discuss the objection of Comment [14] about the estimate of the effective path of ASE in the gain medium.

\section{Effective path of ASE and effective lifetime}

In this section we combine the estimates of papers [12,13] for the effective path of ASE in cases with anti-ASE cap and without (Fig. 1).

The bouncing ray, reflecting at the angle of total internal reflection, may have length of order of $2 L$; such a ray is shown in left

\footnotetext{
* Corresponding author.

E-mail address: dima@ils.uec.ac.jp (D. Kouznetsov).
}

hand side of Fig. 2. Not all photons of ASE are trapped within the active slab, some of them go up or down and leave the active medium at the first intent; also, some photons are emitted to the direction of the closest edge of the disk, they also have path significantly shorter than $2 L$. For this reason, Comment [14] suggests the estimate, there the effective path is $L / 2$, i.e., one quarter of the maximal path shown in Fig. 2a. Due to the exponential growth of ASE, the effective path is determined by the longest rays, not shortest ones; even if majority of rays indeed have length of order of $L / 2$. The more detailed analysis could be performed using ray tracing. Up to our knowledge, such analysis is not yet reported; so, the correction factor for the effective length is not justified. Therefore, we use $L$ without coefficients as the estimate of the effective path of ASE in the gain medium.

Assume that the excitation of the active medium is dropped by ASE in such a way, as if all ASE photons would have some equivalent effective path in the gain medium. This leads to the estimate of the effective lifetime for the disk laser without cap

$\tau=\tau_{0} \exp (-G L)$.

At the anti-ASE cap, the most of photons leave the active layer at the first pass (Fig. 2), and the maximal path of ASE in the active layer is only $\sqrt{2} L$, instead of $2 L$ of uncovered disk. The only small portion of photons, proportional to the angle $2 h / L$, may get significant gain (Fig. 2b). This allows to increase gain $G$ or size $L$ in compare to the uncovered layer, and operate the laser at larger values of the transverse-trip gain $u=G L$. In the case with cap, the maximal length of the ASE ray in the gain medium shorter $(L \sqrt{2}$ instead of $2 L$ ), but the suppression of ASE allows the efficient operation at larger values of the transverse-trip gain $u=G L$, This makes contribution of longest rays even more dominant; it is not obvious, how should we correct the estimate of the effective length, should it be increased or reduced in compare to the case of uncovered disk. Therefore, for the qualitative consideration, we keep $L$ as the 

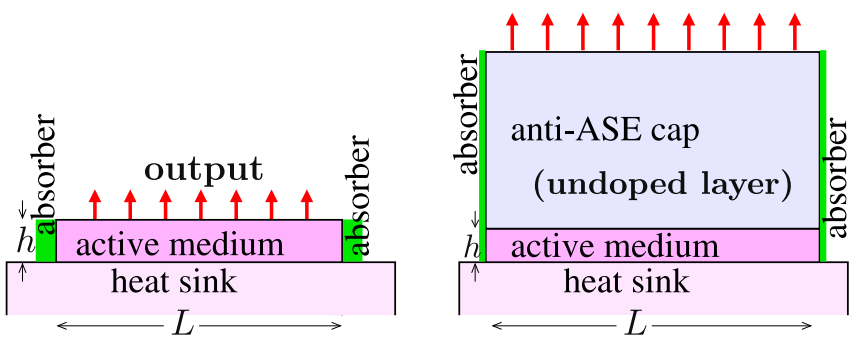

Fig. 1. Uncovered disk laser (left) and that with anti-ASE cap (right).
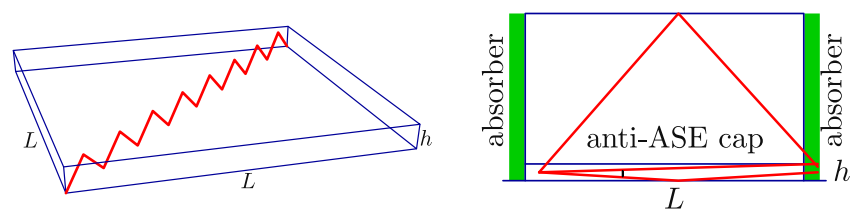

Fig. 2. Left: A path of photons of ASE in a laser without cap: the most of them are trapped in the active slab, the extremal case of a ray of length $2 L$ bouncing along the diagonal in a block of active medium $L \times L \times h$ with total internal reflection is shown. Right: The paths of ASE in a laser with the anti-ASE cap; the maximal length is only $\sqrt{2} L$, and the only spontaneous photons emitted into a solid angle $2 \pi \frac{2 h}{L}$ may get the strong amplification.

estimate of the effective length in both cases, for uncovered disk laser and for the disk with undoped cap. Then, the effective lifetime $\tau$ for the laser disk with anti-ASE cap can be estimated with

$\frac{1}{\tau}=\frac{1}{\tau_{0}}+\frac{h}{L} \frac{\exp (G L)}{\tau_{0}}$.

The estimate of the effective length (and the effective lifetime) above assumes, that no parasitic laser oscillation occurs within the plane of the gain medium. Such oscillations should be suppressed with absorbing layers, shown in Fig. 1 and right hand side of Fig. 2. Then, within our model, the only different estimates (1) and (2) of the effective lifetime make difference between uncovered disk laser and disk with anti-ASE cap (Fig. 1).

\section{Round-trip gain and the round-trip loss}

For the accurate consideration with geometric optics, one should define distribution of gain $G(x, y, z)$ and the gain along each path $X(a), Y(a), Z(a)$ (parameterized with length $a$ ) is determined with the integral $\int G(Z(a), Y(a), Z(a)) \mathrm{d} a$. For our estimates, we take the round-trip gain $g=2 G h$, assuming, that gain $G$ does not vary much inside the pumped region. After passing the gain medium, intensity $I$ becomes $I \exp (g)$. Assume, the only small part of the energy of the light in the cavity is outputted at each round-trip. The intensity $I$ after the output coupler becomes $(1-\theta) I$.

Assume, the background loss $\beta$ at each round-trip reduces the intensity with factor $(1-\beta)$. For the $\mathrm{cw}$ operation, the round-trip gain $g$ compensates both, the output coupling $\theta$ and the background loss $\beta$. At $\beta \ll 1, \theta \ll 1$, the round-trip gain $g=\beta+\theta$. The loss may be determined by technological limitations; then the gain is determined by the output coupling parameter $\theta$. For optimization at given $\beta$, round-trip gain $g$ is good as independent parameter; and the difference $g-\beta=\theta$ is output coupling.

\section{Thermal loading and overheating}

The power of pump is limited with the thermal shock. The thermal shock parameter [6] can be defined as $R_{\mathrm{T}}=\frac{k \sigma_{\mathrm{T}}(1-v)}{\alpha E}$, where $k$ is thermal conductivity, $\sigma_{\mathrm{T}}$ is maximal tension the material can resist, $\alpha$ is the thermal expansion coefficient, $E$ is the Young's modulus, and $v$ is the Poisson ratio. For the layer of the gain medium placed at the heat sink, the pump power cannot exceed value

$P_{\mathrm{p}, \max }=3 \frac{R_{\mathrm{T}}}{q} \frac{L^{2}}{h}$

where $q \approx 1-\frac{\omega_{s}}{\omega_{p}}$ is part of the absorbed pump power which is converted to the heat inside the active medium.

Even if the medium resists well the gradient of the temperature, the raise of temperature at which it still can efficiently operate is limited; assume, the maximal raise $\Delta T$ of temperature is allowed. It limits the pump power with

$P_{\mathrm{p}, \max }=2 \frac{k \Delta T}{q} \frac{L^{2}}{h}$.

The combination of the two estimates (3) and (4) gives the estimate

$P_{\mathrm{p}, \max }=R \frac{L^{2}}{h}$,

where

$R=\min \left\{\begin{array}{c}3 R_{\mathrm{T}} / q \\ 2 k \Delta T / q\end{array}\right.$

is thermal loading parameter [12].

The "abrupt" combination (6) of effects of overheating and of the thermal shock into a single thermal loading $R$ is suitable for qualitative estimates. Generally, in this paper we consider only unavoidable mechanisms, which are essential for the limit of power due to overheating, threshold and ASE. We do not include into this model any effect which "may affect the resulting estimate" [14-30]; but we include an effect if its neglecting does not allow to estimate the upper limit of power. The smooth drop of efficiency at the increase of temperature of the gain medium may be especially important for the detailed model of $\mathrm{Yb}$ doped crystals, where the various sources of thermal load may be equally important $[1,2]$. In this paper we do not analyze these effects and describe the thermal properties of the medium with single parameter $R$. This parameter determines how much pump power can be absorbed in the active medium at given $h$ and $L$.

\section{Threshold}

For the efficient operation, the laser should work well above the threshold. The output power can be estimated with

$P_{\mathrm{s}}=\frac{\omega_{\mathrm{s}}}{\omega_{0}}\left(1-\frac{g}{\beta}\right)\left(P_{\mathrm{p}}-P_{\mathrm{th}}\right)$

where the threshold power $P_{\text {th }}=\frac{h \omega_{p}}{\tau} \frac{g L^{2}}{2 \sigma}[22,12]$.

Define the saturation intensity

$Q=\frac{h \omega_{\mathrm{p}}}{2 \tau_{0} \sigma}$.

Then

$P_{\mathrm{s}}=\eta_{o}\left(1-\frac{g}{\beta}\right)\left(P_{\mathrm{p}}-Q g L^{2} \frac{\tau_{0}}{\tau}\right)$.

This expression can be maximized under condition $P_{\mathrm{p}} \leqslant R L^{2} / h$, assuming Eq. (1) for the uncovered laser and (2) for the laser with cap. It is important to understand, how many parameters do we really need to play with, optimizing the output power. The consideration becomes simple, if we use $g=2 G h, u=G L$ as independent parameters, and express optimized sized $L$ and $h$ in terms of $g$ and $u$.

The condition of the efficient cooling gives: $\frac{L^{2}}{h}=\frac{P_{\mathrm{p}}}{R}$. Condition for gain gives: $\frac{L}{h}=\frac{2 u}{g}$. Then

$L=\frac{g P_{\mathrm{p}}}{2 u R}, \quad h=\frac{g^{2} P_{\mathrm{p}}}{4 u^{2} R}$ 
These relations hold for both uncovered disk laser and the disk with anti-ASE cap, and allow to recover physical dimensions of the optimized laser from parameters $g$ and $u$. The output power also can be expressed in terms of these parameters

$P_{\mathrm{s}}=\frac{\omega_{\mathrm{s}}}{\omega_{0}}\left(1-\frac{\beta}{g}\right)\left(P_{\mathrm{p}}-\frac{P_{\mathrm{p}}^{2} Q}{R^{2}} \frac{g^{3}}{4 u^{2}} \mathrm{e}^{u}\right), \quad$ (no cap),

$P_{\mathrm{s}}=\eta_{0}\left(1-\frac{\beta}{g}\right)\left(P_{\mathrm{p}}-\frac{P_{\mathrm{p}}^{2} Q}{R^{2}} \frac{g^{3}}{4 u^{2}}\left(1+\frac{g}{2 u} \mathrm{e}^{u}\right)\right), \quad$ (with cap).

Within this model, the key parameter is

$P_{\mathrm{k}}=\frac{\omega_{\mathrm{s}}}{\omega_{0}} \frac{R^{2}}{Q \beta^{3}}=\frac{\omega_{\mathrm{s}}}{\omega_{\mathrm{p}}} \frac{R^{2}}{Q \beta^{3}}$.

As $g$ should remain larger than $\beta$, and $\mathrm{e}^{u} / u^{2}$ has minimum, the $P_{\mathrm{k}}$ determines the order of magnitude of the maximal power, achievable at given round-trip loss $\beta$. In the following sections, we consider optimization of the output power for laser without cap and laser with anti-ASE cap, each for two cases, with limited input power and $\left(P_{\mathrm{p}}\right.$ is fixed parameter) and without such a limit (when the pump power is one of optimizing parameters).

In the following, we use dimension-less variables $p$ and $s$ instead of power of pump and that of signal.

$p=P_{\mathrm{p}} / P_{\mathrm{d}}, \quad s=\frac{\omega_{\mathrm{p}}}{\omega_{\mathrm{s}}} P_{\mathrm{s}} / P_{\mathrm{d}}, \quad P_{\mathrm{d}}=R^{2} / Q$

for each of the four cases (given $p$ or maximal $p$ for the disk with- or without cap).

\section{Case without cap, optimization at given $p$}

For the case without cap at given $p$, we have

$s=\left(1-\frac{\beta}{g}\right)\left(p-p^{2} \frac{g^{3} \mathrm{e}^{u}}{4 u^{2} \beta^{3}}\right)$
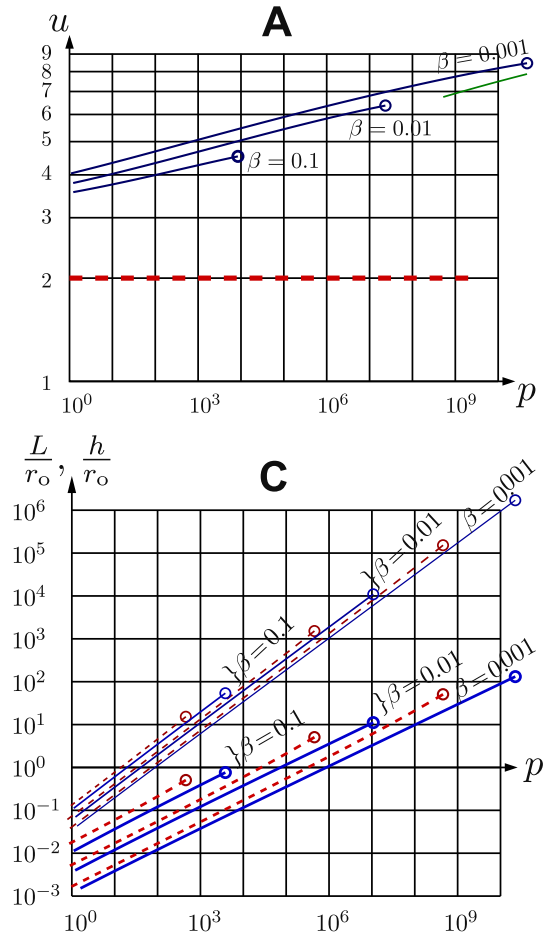

This expression has maximum at $u=2$; this level is shown in Fig. 3A with dashed horizontal line. Then we find the optimal round-trip gain, maximizing

$s=\left(1-\frac{\beta}{g}\right)\left(p-p^{2} \frac{g^{3} \mathrm{e}^{2}}{16 \beta^{3}}\right)$.

The maximization of $s$ with respect to $g$ gives

$\frac{16 / \mathrm{e}^{2}}{3-2 \beta / g}\left(\frac{\beta}{g}\right)^{4}=\beta^{3} p$.

The resulting gain $g$ versus $p$ is shown in Fig. 3B with dashed lines.

Graphics in Fig. 3 look almost straight. This can be interpreted as scaling laws. For the efficient operation $(g \gg \beta)$, we have an estimate

$g \approx\left(\frac{16 \beta}{3 \mathrm{e}^{2}}\right)^{1 / 4} p^{-1}$.

Together with condition $u=2$ and Eq. (10), estimate (18) determines also the simple expressions for scaling of optimal sizes $L$ and $h$ at the power scaling.

As the power approaches the maximum, the approximation $g \gg \beta$ is not valid and the graphics deviate from straight lines. This deviation is small. For $\beta=0.001$, the approximation (18) is shown with thin dashed line at the bottom of Fig. 3B. At logarithmic scale, this line almost coincides with the thick dashed curve, representing the solution of Eq. (17).

The optimal sizes $L$ and $h$ can be estimated from (10); these sizes are shown in Fig. 3C with dashed lines for various values of $\beta$; they also look almost straight. In order to make the result not dependent on the particular medium, dimension-less variables $L / r_{0}$ and $h / r_{0}$ are used; $r_{0}=R / Q$. The resulting normalized output power is shown in Fig. 3C.

Fig. 3D shows, that at each $\beta$, there is some maximal pump power; the optimization for a larger pump power per active element does not lead to an increase of the output power. If we are interested in the maximal power per active element and do not

B
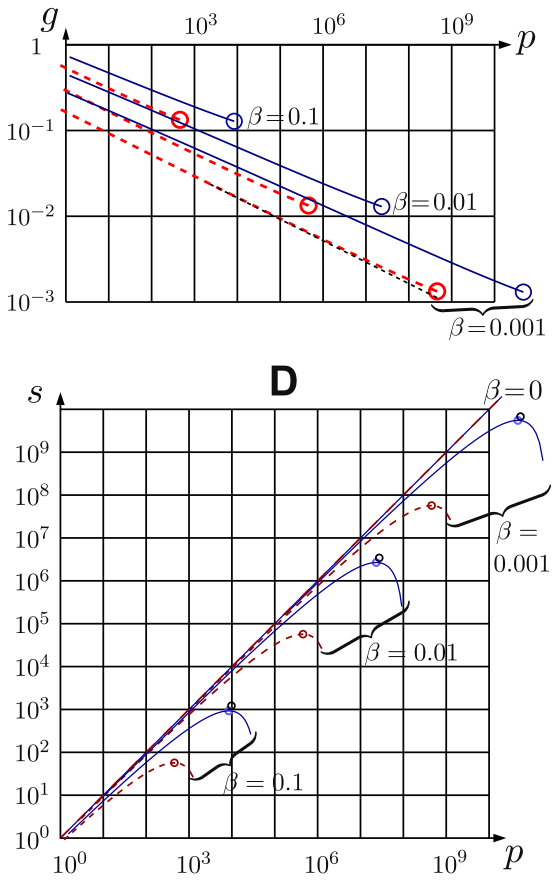

Fig. 3. Optimized parameters $u$, g, sizes $L$ and $h$ at $\beta=0.1,0.01,0.001$ and corresponding normalized power $s=P_{\mathrm{p}} / P_{\mathrm{d}}$ achievable versus $p=P_{\mathrm{p}} / P_{\mathrm{d}}$ for uncovered disk laser (dashed) and that with anti-ASE cap (solid). 
care about efficiency, we consider $p$ as variable. Optimal $u=2$ keeps as in previous case, but now optimal round-trip gain $g=\frac{4}{3} \beta$. This gives optimal sizes

$L=L_{\max }=\frac{r_{0}}{8 \mathrm{e}^{2}}\left(\frac{3}{\beta}\right)^{2}, \quad h=h_{\max }=\frac{r_{0}}{8 \mathrm{e}^{2}} \frac{3}{\beta}$

optimal power of pump and corresponding power of signal are

$p=p_{\max }=\frac{1}{8 \mathrm{e}^{2}}\left(\frac{3}{\beta}\right)^{3} ; \quad s=s_{\max }=\frac{1}{64 \mathrm{e}^{2}}\left(\frac{3}{\beta}\right)^{3}$.

At such a maximal power, the efficiency is low; it does not exceed $1 /$ 8. If the disk laser works close to the limit of the power scaling, then significant part of the pump power is absorbed in the absorbing layers (Figs. 1 and 2b). In order to deliver this power to the heat sink, the absorbing layer should be thick. High density of ASE allows its recycling: the absorbing layer might be also an active medium that can be efficiently pumped at frequency $\omega_{\mathrm{s}}$. Such a co-laser could help to drain the unusable energy from the edges of the disk laser and simplify the cooling. The development of laser materials with shifted working frequencies of pump and signal may found application in powerful disk lasers.

\section{Optimization with cap}

For the case with anti-ASE cap, the dimension-less output power $s=\frac{\omega_{\mathrm{s}}}{\omega_{\mathrm{p}}} P_{\mathrm{s}} / P_{\mathrm{d}}$ can be estimated as follows:

$s=\left(1-\frac{\beta}{g}\right)\left(p-\frac{p^{2} g^{3}}{4 u^{2}}\left(1+\frac{g}{2 u} \mathrm{e}^{u}\right)\right)$.

We set the derivatives with respect to $u$ and $g$ to zero; this gives the equations

$\frac{4 u \mathrm{e}^{-u}}{u-3}=g, \quad \frac{2 g^{4} p\left(2 \mathrm{e}^{u} g+3 u\right)}{3 \mathrm{e}^{u} g^{4} p+4 g^{3} p u+8 u^{3}}=\beta$,

leading to

$\frac{(u-3)^{5} \mathrm{e}^{4 u} \beta}{32 u^{2}\left(6 u-2-\beta u \mathrm{e}^{u}(u-3)\right)}=p$.

We may use $u$ for parameterization of graphics; then we have no need to solve any transcendental equation in order to plot optimal $u$ versus $p$ at given $\beta$.

As in the case without cap, there is maximal pump power; we maximize (21) with respect to $u, g$ and $p$.

Maximization with respect to $p$ gives

$p=\frac{4 u^{3}}{g^{3}\left(\mathrm{e}^{u} g+2 u\right)}, \quad s=\frac{2(g-\beta) u^{3}}{g^{4}\left(\mathrm{e}^{u} g+2 u\right)}$.

The maximization with respect to $u$ and $g$ gives

$\frac{4 \mathrm{e}^{-u} u}{u-3}=g, \quad \frac{2 g\left(\mathrm{e}^{u} g+3 u\right)}{5 \mathrm{e}^{u} g+8 u}=\beta$.

The combination gives

$\frac{2 u \mathrm{e}^{-u}(3 u-1)}{(2 u-1)(u-3)}=\beta$

Again, no need to solve a transcendental equation in order to plot optimal $u$ versus $\beta$.

As the consideration is qualitative, the simple asymptotic estimates is more suitable than the exact solution of approximate equations. We re-write the Eq. (26) as follows: $u=\ln \frac{3}{\beta}+\ln \left(1-\frac{1}{3 u}\right)-\ln \left(1-\frac{1}{2 u}\right)-\ln \left(1-\frac{3}{u}\right)$. We see, new variable

$\varepsilon=\frac{1}{\ln (3 / \beta)}$ can be used as small parameter; then, optimal transverse-trip gain

$u \approx \frac{1}{\varepsilon}+\frac{19}{6} \varepsilon+\mathcal{O}\left(\varepsilon^{2}\right)$.

For $\beta=0.001$, the leading term of this asymptotic is shown in Fig. 3A with thin solid line; it is close to the thick line, which represents more accurate optimization. This indicates that the use of only leading terms of asymptotic in this section gives reasonable approximation.

Using this the asymptotic (28), we estimate the optimal roundtrip gain

$g \approx \frac{4}{3} \beta\left(1+\frac{1}{6} \varepsilon+\mathcal{O}\left(\varepsilon^{2}\right)\right)^{-1}$

and optimal sizes

$L \approx \frac{r_{0}}{16}\left(\frac{3}{\beta \varepsilon}\right)^{2}\left(1+\frac{5}{3} \varepsilon+\mathcal{O}\left(\varepsilon^{2}\right)\right)^{-1}$

$h \approx \frac{r_{0}}{8}\left(\frac{3}{\beta}\right)\left(1+\frac{11}{3} \varepsilon+\mathcal{O}\left(\varepsilon^{2}\right)\right)^{-1}$

The maximal power is estimated with

$s \approx\left(\frac{3}{\beta}\right)^{3} \frac{1}{256 \varepsilon^{2}}\left(1+2 \varepsilon+\mathcal{O}\left(\varepsilon^{2}\right)\right)^{-1}$

$p \approx\left(\frac{3}{\beta}\right)^{3} \frac{1}{32 \varepsilon^{2}}\left(1+\frac{3}{2} \varepsilon+\mathcal{O}\left(\varepsilon^{2}\right)\right)^{-1}$

The asymptotical estimates above are written in such a way, that the partial sums remain positive (and have physical sense) even at $\varepsilon \approx 1$. The leading terms of the estimates are shown with thin circles in Fig. 3D; at the logarithmic scale, they look very close to the thick circles, which correspond to exact maxima of expression (21); the leading terms in asymptotic in this section give good approximation for the optimized parameters of the thin disk laser. The simplicity of these expressions means that again, as in the case of uncovered disk, we have the scaling laws.

At small values of $\beta$, the ratio of maximal power of a disk laser with cap to that with uncovered active layer can be expressed as follows:

$\frac{\text { power with cap }}{\text { power of uncovered disk }} \approx \frac{\frac{1}{256 \varepsilon^{2}}\left(\frac{3}{\beta}\right)^{3}}{\frac{1}{64 \mathrm{e}^{2}}\left(\frac{3}{\beta}\right)^{3}}=\frac{\mathrm{e}^{2}}{4 \varepsilon^{2}}=\frac{\mathrm{e}^{2}}{4} \ln \left(\frac{3}{\beta}\right)^{2}$

at $\beta \approx 0.01$, at the same round-trip loss $\beta$, anti-ASE cap may allow to increase the maximal output power. However, the practical application of the anti-ASE cap should depend also on the affect of this cap on the round-trip loss, or on the ability to manufacture a cap which, does not increase the round-trip loss. Such a cap may allow to increase the size $L$ to centimeters. The manufacturing of a single crystal becomes difficult with the increase of the size, while the optical properties of modern ceramics are very similar to those of single crystals $[29,30]$. Therefore, the application of the anti-ASE cap refers to the ceramic technology rather than to the growth of a wide single crystal. The scaling laws (28)-(33), as well as the scaling laws (10), (19), (20) from the previous sections have sense while the power of laser is limited mainly by the ASE, background loss and thermal loading. While a disk laser operates far from the maximal power achievable at the given round-trip loss $\beta$, other mechanisms (not taken into account here) may affect the power scaling; but the output power cannot exceed the estimate (20) for uncovered disk and estimate (32) for a thin disk laser with anti-ASE cap. Such a statement is general and needs to be confirmed (or negated) with experimental data.

\section{Comparison with experimental data, recovery of $\beta$}

We do not count with original data about the thermal loading $R$ and round-trip loss $\beta$ in disk lasers of highest power. Therefore, we 
write all the estimate in dimension-less form, for the normalized variables. Such estimates have no need to be revised each time when a breakthrough experiment suggests significant improvement of properties of a laser material: increase of $R$ or reduction of $\beta$. Nevertheless, we should deal with dimensional variables in order to compare our estimates with the experimental data. We suggest such a comparison in this section.

Usually, no estimate of $\beta$ is reported in the experimental papers. Instead, the output coupling $\theta$ is specified (The round-trip gain is determined with $g=\theta+\beta$ ). Also, many authors specify the efficiency $\eta_{\mathrm{a}}$ of absorption of pump and the slope efficiency $\eta_{\mathrm{s}}$ achieved; it can be expressed also with

$\eta_{s}=\frac{\omega_{\mathrm{s}}}{\omega_{0}} \eta_{\mathrm{a}} \frac{\theta}{\theta+\beta}$

Form this equation, we get the estimate for the round-trip loss

$\beta=\theta\left(\frac{\omega_{\mathrm{s}}}{\omega_{0}} \frac{\eta_{\mathrm{a}}}{\eta_{\mathrm{s}}}-1\right)$

used to construct the Table 1 . The data from Table 1 are shown in Fig. 4. Estimates (20) and (32) of maximal power are very sensitive to the round-trip loss $\beta$. The estimates of maximal $\beta$, at which the desired normalized output power $s$ can be achieved, are robust. Therefore, in Fig. 4, $\beta$ versus $s$ is plotted.

Although the estimate of $\beta$ and $R$ in Table 1 are rather guess than a well measured values, the resulting Fig. 4 shows that our estimate does not contradict experimental data. All the experimental data (even those reported after the publication [12] of the estimate (20)) are below the theoretical upper bound. Any datum with round-trip loss $\beta$ above the theoretical estimate in Fig. 4 would contradict our model; up to our knowledge, no such data are reported. The use of dimension-less variable instead of output power allows to extend our predictions to wide class of disk lasers, including those which are not yet reported. In such a way, our prediction applies to any thin disk laser, with any medium which allows to determine the thermal loading $R$ and saturation intensity $Q$.

The quality of a laser medium is not characterized by the position of the laser at the diagram in Fig. 4. The appropriate choice of wavelength of pump and that of signal (or even use of a new very prospective medium which is not yet reported) may reduce the quantum defect (especially at cryogenic temperatures), increasing $R$ and reducing $Q$. This would increase the key parameter $P_{\mathrm{k}}$ and allow to increase the output power (even at same round-trip loss $\beta$ ). Such an advance has no need to increase the normalized power $s$; the experimental dots (being plotted in Fig. 4) are expected to remain at the lower side of the graphic.

The correction coefficient $1 / 2$ in the estimate for the effective length of ASE in the gain medium is suggested by [14]. Such a correction would lead to a factor 4 in the estimate for the maximal power, displacing the curves to right hand side for more than a half of the step of the grid. Such a correction would lead to a safer (softer, weaker) estimate. There is nothing wrong in application of a correction coefficient to the estimates, obtained with qualitative

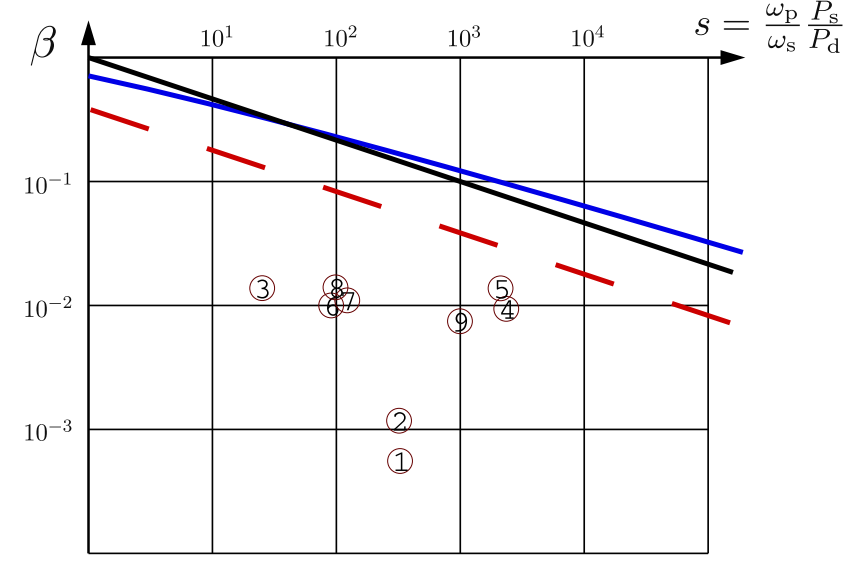

Fig. 4. Plot of $\beta$ and $s$ from experimental data. Digits in circles indicates the raws in Table 1 . The dashed line, estimate for the disk without cap by (20). The solid curve shows the estimate by (24) and (26) for the disk with cap. The straight solid line represents the rough estimate $\beta=p^{-1 / 3}$ without coefficients.

analysis above. However, until now, no experimental data indicate any need in such a correction; all the results are at least one order of magnitude left from the limit, marked with dashed line in Fig. 4. Our estimate of the lower bound for the round-trip loss (or the estimate of the maximal power at given round-trip loss $\beta$ ) does not contradict the experimental data.

The key parameter $P_{\mathrm{k}}$ by (13) is criterion for comparison of different gain media. Parameters $R$ and especially $\beta$ are difficult to recover from publications about experimental realizations of disk lasers, and the estimate with Eq. (35) is not accurate. This estimate may indicate properties of the $\mathrm{Yb}: \mathrm{Lu}_{2} \mathrm{O}_{3}$, which allow to prepare the surfaces with high quality and achieve especially low values of the round-trip loss $\beta$. However, the value of $P_{\mathrm{k}}$ in the upper right corner of the Table 1 is so huge, that it can be attributed, at least partially, to the poor recovery of $\beta$ with Eq. (35). Careful measurement of parameters $R$ and $\beta$ for various laser materials is important for their evaluation. Especially important are estimates of parameter $P_{\mathrm{k}}$ for laser ceramics, which allow to make a thin wide slab of the active medium.

\section{Conclusions}

Two configurations (Fig. 1) are analyzed within simple model. The laser material is characterized with thermal loading $R$, saturation intensity $Q$ and background loss $\beta$. This model reveals the scaling laws for the optimal parameters of such a laser at the power scaling and allows to estimate the maximal power achievable at given round-trip loss $\beta$. The upper limit of power presented here should be compared to the limits due to other mechanisms.

The upper limit of power is consistent with published experimental data (Fig. 4). The thickness $h$ and size $L$, which allow to achieve the maximal power, follow the scaling laws $h \sim \frac{R}{Q \beta}$,

Table 1

$\beta$ and $s$ from experimental data

\begin{tabular}{|c|c|c|c|c|c|c|c|c|c|c|c|c|c|c|c|c|}
\hline Material $\backslash$ unit & $h(\mathrm{~nm})$ & $\lambda_{\mathrm{p}}(\mathrm{nm})$ & $\lambda_{\mathrm{s}}(\mathrm{nm})$ & $R \frac{\text { Watt }}{\mathrm{mm}}$ & $Q \frac{\mathrm{kW}}{\mathrm{cm}^{2}}$ & $\frac{\omega_{\mathrm{s}}}{\omega_{0}}(\%)$ & $\theta(\%)$ & $\eta_{\mathrm{a}}(\%)$ & $\eta_{\mathrm{s}}(\%)$ & $P_{\mathrm{s}}$ (Watt) & Cite & $P_{\mathrm{d}}$ (Watt) & $\beta(\%)$ & $s$ & Raw number & $P_{\mathrm{k}}(\mathrm{MW})$ \\
\hline 03\%Yb:Lu2O3 & 0.25 & 976 & 1080 & 8 & 42 & 90.40 & 0.40 & 95.0 & 75.4 & 33 & [23] & 0.2 & 0.06 & 237 & 1 & 886.71 \\
\hline 25\%Yb:Lu2O3 & 0.25 & 976 & 1080 & 8 & 42 & 90.40 & 1.60 & 95.0 & 80.0 & 32 & [23] & 0.2 & 0.12 & 232 & 2 & 93.69 \\
\hline 19\%Yb:Lu2O3 & 0.25 & 976 & 1034 & 14 & 13 & 94.40 & 5.70 & 95.0 & 72.2 & 26 & [23] & 1.5 & 1.38 & 18 & 3 & 0.57 \\
\hline 10\%Yb:LSB & 0.30 & 974 & 1040 & 3 & 36 & 94.00 & 1.00 & 99.0 & 48.0 & 40 & [24] & 0.0 & 0.94 & 1702 & 4 & 0.03 \\
\hline 08\%Yb:LSB & 0.30 & 974 & 1040 & 3 & 36 & 94.00 & 1.00 & 96.0 & 38.0 & 36 & [24] & 0.0 & 1.37 & 1532 & 5 & 0.01 \\
\hline 08\%Yb:YAG & 0.25 & 941 & 1030 & 20 & 5 & 91.20 & 2.20 & 0.0 & 56.0 & 480 & [25] & 8.0 & 1.00 & 66 & 6 & 8.00 \\
\hline 09\%Yb:YAG & 0.25 & 941 & 1030 & 20 & 5 & 91.20 & 3.00 & 0.0 & 60.0 & 647 & [25] & 8.0 & 1.10 & 89 & 7 & 6.01 \\
\hline 10\%Yb:YAG & 0.20 & 940 & 1030 & 20 & 5 & 91.20 & 3.00 & 0.0 & 56.0 & 520 & [26] & 8.0 & 1.40 & 71 & 8 & 2.92 \\
\hline Yb:YAG & 0.10 & 940 & 1030 & 20 & 5 & 92.00 & 3.00 & 95.0 & 70.0 & 5000 & {$[27,28]$} & 7.5 & 0.75 & 720 & 9 & 18.20 \\
\hline
\end{tabular}


$L \sim \frac{R}{Q \beta^{2}}$. The key parameter $P_{\mathrm{k}}=\frac{\omega_{\mathrm{s}}}{\omega_{\mathrm{p}}} \frac{R^{2}}{Q \beta^{3}}$ determines the limit of power. Evaluation of this parameter using estimate (36) indicates advantages of $\mathrm{Yb}: \mathrm{Lu}_{2} \mathrm{O}_{3}$, although the huge value of $R_{\mathrm{k}}$ in Table 1 can be attributed also to the qualitative character of the estimate (36) for the round-trip loss $\beta$. Systematic measurement of $\beta, R$ and comparison of resulting $P_{\mathrm{k}}$ is important for evaluation of laser materials, and especially laser ceramics.

The key parameter $P_{\mathrm{k}}$ remains important at the use of the antiASE cap (Fig. 1). The anti-ASE cap allows to increase the maximal power with factor $\sim(\ln \beta)^{2}$, assuming the same value of $\beta$. This might give an additional order of magnitude to the maximal power, achievable with given laser material. While the reported lasers did not yet reach the limit of power scaling (Fig. 4), the application of such a cap should depend on its affect on other parameters, in particular, the round-trip loss $\beta$.

\section{Acknowledgement}

Authors are grateful to Jun Dong, Jian Lang Li, Olivier Parriaux and Robert Byer for the discussions. This work was supported by the 21st Century COE program of Ministry of Education, Science and Culture of Japan.

\section{References}

[1] K. Ueda, N. Uehara, Laser-diode-pumped solid state lasers for gravitational wave antenna, in: Proceedings of SPIE 1837, 1993, pp. 336-345.

[2] A. Giesen, H. Hügel, A. Voss, K. Wittig, U. Brauch, H. Opower, Scalable concept for diode-pumped high-power solid-state lasers, Appl. Phys. B 58 (5) (1994) 365-372.

[3] C. Li, D.Y. Shen, J. Song, N.S. Kim, K. Ueda, Theoretical and experimental investigations of diode-pumped Tm: YAG laser in active mirror configuration, Opt. Rev. 6 (5) (1999) 439-442.

[4] N.P. Barnes, B.M. Walsh, Amplified spontaneous emission - application to Nd:YAG lasers, IEEE J. Quant. Electron. 35 (1) (1999) 101-109; N.P. Barnes, B.M. Walsh, Corrections to Amplified spontaneous emission application to Nd:YAG lasers, IEEE J. Quant. Electron. 35 (7) (1999) 1100.

[5] C. Stewen, K. Contag, M. Larionov, A. Giesen, H. Hügel, A 1-kW CW thin disc laser, IEEE J. Selected Topics Quant. Elect. 6 (4) (2000) 650-657.

[6] R.J. Beach, E.C. Honea, C. Bibeau, S.A. Payne, H. Powell, W.F. Krupke, S.B. Sutton, High average power scalable thin-disk laser. US patent 6,347,109, February 12, 2002.

[7] L.E. Zapata, R.J. Beach, E.C. Honea, S.A. Payne, Method for optical pumping of thin disk laser media at high average power. USA patent No.: US 6,763,050, 2004.

[8] K. Naito, M. Yamanaka, M. Nakatsuka, T. Kanabe, K. Mima, C. Yamanaka, S. Nakai, Conceptual design of a laser diode pumped solid state laser system for laser fusion reactor driver, Jpn. J. Appl. Phys. 31 (2A) (1992) 259-273.
[9] C.D. Orth, S.A. Payne, W.F. Krupke, A diode pumped solid state laser driver for inertial fusion energy, Nucl. Fusion 36 (1) (1996) 75-116.

[10] E.I. Moses, C.R. Wuest, The national ignition facility: laser performance and first experiments, Fusion Sci. Technol. 47 (3) (2005) 314-322.

[11] E. Innerhofer, T. Südmeyer, F. Brunner, R. Häring, A. Aschwanden, R. Paschotta, C. Hönninged, M. Kumkar, U. Keller, $60-\mathrm{W}$ average power in $810-$ fs pulses from a thin-disk Yb:YAG laser, Opt. Lett. 28 (5) (2003) 367-369.

[12] D. Kouznetsov, J.-F. Bisson, J. Dong, K. Ueda, Surface loss limit of the power scaling of a thin disk laser, J. Opt. Soc. Am. B 23 (2006) 1074-1082.

[13] D. Kouznetsov, J.-F. Bisson, Role of undoped cap in the scaling of thin disk lasers, J. Opt. Soc. Am. B 25 (2008) 338-345.

[14] R. Paschotta, J. Speiser, A. Giesen, Comment on Surface loss limit of the power scaling of a thin-disk laser, J. Opt. Soc. Am. B 24 (2007) 2658.

[15] D. Kouznetsov, J.-F. Bisson, K. Takaichi, K. Ueda, High-power single-mode solid-state laser with short, wide unstable cavity, J. Opt. Soc. Am. B 22 (2005) 1605-1619.

[16] N. Uehara, A. Ueda, K. Ueda, H. Sekiguchi, T. Mitake, K. Nakamura, N. Kitajima, I. Kataoka, Ultralow-loss mirror of the parts-in- $10^{6}$ level at $1064 \mathrm{~nm}$, Opt. Lett. 20 (6) (1995) 530-532.

[17] W.F. Krupke, M.D. Shinn, J.E. Marion, J.A. Caird, S.E. Stokowski, Spectroscopic, optical, and thermomechanical properties of neodymium- and chromiumdoped gadolinium scandium gallium garnet, J. Opt. Soc. Am. B 3 (1) (1986) $102-114$

[18] T.Y. Fan, Heat generation in Nd:YAG and Yb:YAG, IEEE J. Quant. Electron. 29 (6) (1993) 1457-1459.

[19] T. Kasamatsu, H. Sekita, Y. Kuwano, Temperature dependence and optimization of $970 \mathrm{~nm}$ diode-pumped Yb:YAG and Yb:LuAG lasers, Appl. Opt. 38 (24) (1999) 5149-5153.

[20] J. Lu, J. Lu, K. Takaichi, T. Uematsu, K. Ueda, H. Yagi, T. Yanagitani, A.A. Kaminskii, $\mathrm{Nd}^{3+}: \mathrm{Y}_{2} \mathrm{O}_{3}$ ceramic laser, Jpn. J. Appl. Phys. 40 (Part 2) (2001) L1277-L1279.

[21] Q. Liu, M.L. Gong, Y.Y. Pan, C. Li, Edge-pumped composite thin-disc Yb:YAG/ YAG laser: design and power scaling, Acta phys. Sin. 53 (7) (2004) 2159-2164.

[22] B. Henderson, R.H. Bartram, Crystal-field engineering of solid-state laser materials, Cambridge University Press, 2000

[23] R. Peters, C. Kränkel, K. Petermann, G. Hüber, Broadly tunable high-power $\mathrm{Yb}: \mathrm{Lu}_{2} \mathrm{O}_{3}$ thin disk laser with $80 \%$ slope efficiency, Opt. Express 15 (11) (2007) 78075-78082.

[24] C. Kränkel, J. Johannsen, R. Peters, K. Peterman, G. Huber, Continuous wave high power laser operation and tunability of $\mathrm{Yb}: \mathrm{LaSc}_{3}\left(\mathrm{BO}_{3}\right)_{4}$, Appl. Phys. B 87 (2007) 217-220.

[25] C. Stewen, K. Contag, M. Larionov, A. Giesen, H. Huegel, A 1-kW CW thin disc laser, IEEE J. Selec. Top. Quant. Electron. 6 (4) (2000) 450-657.

[26] M. Tsunekane, T. Taira, High-power operation of diode edge-pumped, composite all-ceramic $\mathrm{Yb}: \mathrm{Y}_{3} \mathrm{Al}_{5} \mathrm{O}_{12}$ microchip laser, Appl. Phys. Lett. 90 (2007) 121101

[27] A. Giesen, J. Speiser, R. Peters, C. Kränkel, K. Peterman, Thin-disk lasers come of age. Photonics Spectra (May) (2007) 52-58.

[28] A. Giesen, J. Speiser, Fifteen years of work on thin-disk lasers: results and scaling laws, IEEE J. Select. Topics Quant. Electron. 13 (2007) 598-609.

[29] J. Lu, H. Yagi, K. Takaichi, T. Uematsu, J.-F. Bisson, Y. Feng, A. Shirakawa, K.I. Ueda, T. Yanagitani, A.A. Kaminskii, $110 \mathrm{~W}$ ceramic $\mathrm{Nd}^{3+}: \mathrm{Y}_{3} \mathrm{Al}_{5} \mathrm{O}_{12}$ laser, J. Appl. Phys. B 79 (2004) 25-28.

[30] Y. Qi, X. Zhu, Q Lou, J. Ji, J. Dong, R. Wei, High-energy LDA side-pumped electro-optical Q-switched Nd:YAG ceramic laser, J. Opt. Soc. Am. B 24 (2007) 1042-1045 